\title{
New dynamic programming algorithms for the resource-constrained elementary shortest path problem
}

\author{
Giovanni Righini, Matteo Salani* \\ Dipartimento di Tecnologie dell'Informazione \\ Università degli Studi di Milano \\ Via Bramante 65, 26013 Crema, Italy
}

January 2005

\begin{abstract}
The resource-constrained elementary shortest path problem arises as a pricing subproblem in branch-and-price algorithms for vehicle routing problems with additional constraints. We address the optimization of the resource-constrained elementary shortest path problem and we present and compare three methods. The first method is a well-known exact dynamic programming algorithm improved by new ideas, such as bi-directional search with resource-based bounding. The second method consists of a branch-and-bound algorithm, where lower bounds are computed by dynamic programming with state space relaxation; we show how bounded bi-directional search can be combined with state space relaxation and we present different branching strategies and their hybridization. The third method, called decremental state space relaxation, is a new one; exact dynamic programming and state space relaxation are two special cases of this new method. The experimental comparison of the three methods is definitely favourable to decremental state space relaxation. Computational results are given for different kinds of resources, arising from the capacitated vehicle routing problem, the vehicle routing problem with distribution and collection and the vehicle routing problem with capacities and time windows.
\end{abstract}

Keywords: shortest path, vehicle routing, column generation, dynamic programming, branch-and-bound.

\footnotetext{
*Corresponding author: (salani@dti.unimi.it)
} 


\section{Introduction}

Branch-and-price is one of the most effective techniques for the exact optimization of vehicle routing problems (VRP) with additional constraints. At each node of a branch-and-bound tree a relaxation of the set covering reformulation of the problem is solved via column generation. Algorithms based on this technique can solve constrained VRP instances with more than 100 vertices (see for instance Kohl et al. [20]). When vehicle routing problems with additional constraints are solved via column generation and branch-and-price, the pricing problem requires to find a resource-constrained elementary path of minimum cost between two given vertices of a weighted graph, with positive costs on the arcs and non-negative prizes on the vertices. The cost of the path is given by the sum of the costs of the arcs traversed minus the sum of the prizes collected at the vertices visited. Exact optimization is needed to find new columns with negative reduced cost or to prove that none of them exists. For a detailed exposition of branch-and-price methods for vehicle routing problems we refer the reader to Desrosiers et al. [11], Bramel and Simchi-Levi [4] and Cordeau et al. [6].

If the underlying graph may have negative cost cycles (which is the case when there are prizes on the vertices), the resource-constrained elementary shortest path problem (RCESPP) is strongly NP-hard: the proof is due to Dror [13]. The most commonly used technique to solve the RCESPP to optimality is dynamic programming, relying upon the seminal work by Desrochers [7] for the resource-constrained shortest path problem (RCSPP) in which the solution is not required to be elementary. Other methods, based on Lagrangean relaxation, were proposed by Handler and Zang [17] and Beasley and Christofides [3] and were recently examined by Dumitrescu and Boland [14], who devised improved preprocessing and bounding techniques. However these methods require a graph free from negative cost cycles, so that the Lagrangean subproblem is a polynomially solvable shortest path problem. The case with negative cost cycles was considered by Feillet et al. [15], who suggested some improvements to the basic Desrochers' algorithm [7]. For a recent survey on models and algorithms for the RCSPP and the RCESPP we refer the reader to Irnich and Desaulniers [18].

In this paper we consider the problem of computing optimal solutions to the RCESPP, as in Feillet et al. [15], and we present three different approaches: exact dynamic programming, branch-and-bound based on state space relaxation and decremental state space relaxation. All of them use dynamic programming but in different ways: in the first case an exact dynamic programming algorithm computes the optimal solution of the RCESPP; this approach was taken for instance by Feillet et al. [15] and Righini and Salani [22]. In the second case a dynamic programming algorithm with state space relaxation is used to optimize the RCSPP, where cycles are allowed. This gives lower bounds corresponding to non-elementary paths and these lower bounds are exploited in a branch-and-bound framework. The third method, decremental state space relaxation, is original and includes both exact dynamic programming and state space relaxation as special cases. 
The performance of exact dynamic programming algorithms for the RCESPP can be significantly improved through bi-directional search and resource-based bounding, as shown by Righini and Salani [22]. In this paper we review them and we show that they can be applied also to dynamic programming with state space relaxation and decremental state space relaxation. We report on the outcome of experimental comparisons between these methods when solving RCESPPs with different kinds of resource constraints. In particular we consider three variations of the RCESPP arising from three well-known vehicle routing problems with additional constraints, namely the capacitated vehicle routing problem (CVRP), the vehicle routing problem with distribution and collection (VRPDC) and the vehicle routing problem with capacities and time windows (CVRPTW).

The outline of this paper is the following: in Section 2 we formally define the RCESPP; in Section 3 we review the dynamic programming algorithms for its exact optimization; in Section 4 we review bounded bi-directional dynamic programming; in Section 5 we analyze state space relaxation to compute lower bounds; in Section 6 we present the branch-and-bound algorithm; in Section 7 we introduce decremental state space relaxation; in Section 8 we report on computational results; in Section 9 we point out some conclusions. The content of Sections 3 and 4 is a review of concepts already described in [22], which have been recalled here in order to make this paper self-contained.

\section{Problem definition}

The RCESPP is defined as follows: a graph $\mathcal{G}(\mathcal{V}, \mathcal{A})$ is given, where the vertex set $\mathcal{V}$ is made by a set of vertices $\mathcal{N}$ representing $N$ customers and two vertices $s$ and $t$ representing the depot. A non-negative $\operatorname{cost} c_{i j}$ is associated with each arc $(i, j) \in \mathcal{A}$; arc costs correspond to shortest paths and therefore they satisfy the triangle inequality. A non-negative prize $\lambda_{i}$ is associated with each vertex $i \in \mathcal{N}$, a non-negative cost $\lambda_{0}$ is associated with the depot. A vehicle must go from $s$ to $t$, visiting a subset of the other vertices; no cycles are allowed. The objective is to minimize the cost, given by the sum of the costs of the arcs traversed minus the sum of the prizes collected at the vertices visited. In a column generation framework this corresponds to generate columns of minimum reduced cost for the linear relaxation of the set covering reformulation of a VRP: $\lambda_{i}$ is the dual multiplier associated with the covering constraint of vertex $i$ and $\lambda_{0}$ is the dual multiplier asociated with the constraint on the maximum number of available vehicles.

These definitions of the problem are common to all RCESPP versions arising from the different routing problems we consider. Additional constraints, that depend on the kind of vehicle routing problem at hand, are modeled as resource constraints and they are specified hereafter.

Capacity. In the CVRP a non-negative integer demand $d_{i}$ is associated with each vertex $i \in \mathcal{N}$ and a positive integer vehicle capacity $Q$ is given. The sum of the demands of the nodes visited by the same vehicle cannot exceed $Q$. 
Distribution and collection. In the VRPDC each vertex $i$ has two nonnegative integer quantities $p_{i}$ and $d_{i}$ associated with it, representing respectively the amount of load to be collected and to be delivered at that vertex. Each vehicle has a positive integer capacity $Q$, it leaves the depot carrying the total amount of load it must deliver and returns to the depot carrying the total amount of load it has collected. The capacity cannot be exceeded anywhere along the path.

Capacity and time windows. In the CVRPTW a non-negative integer service time $\theta_{i}$ and a time window $\left[a_{i}, b_{i}\right]$, defined by two non-negative integers, are associated with each vertex $i \in \mathcal{N}$ and the service at each visited vertex must start inside its time window. If the vehicle arrives at vertex $i$ before time $a_{i}$, it waits until $a_{i}$. The traveling time from any vertex $i$ to any vertex $j$ is a non-negative integer datum $v_{i j}$.

We chose these three problems, because they offer a significant mix of different characteristics. In the CVRP there is only one resource, whose consumption depends on the vertices visited. In the VRPDC there are two resources associated with the vertices visited and they are interacting: the consumption of one of them also depends on the consumption of the other. In the CVRPTW there are two resources, one associated with the vertices visited and the other associated with the arcs traversed. In all cases resources are subject to a global constraint on their overall consumption along the $s$ - $t$ path, with the exception of the case with time windows, where a resource (time) is subject to local constraints, one for each vertex visited.

\section{Exact dynamic programming}

The starting point for our exposition is the reaching algorithm of Desrochers [7] for the RCSPP, that is an extension of the well-known shortest path algorithm of Ford and Bellman (see [2]). The algorithm assigns states to each vertex: each state associated with vertex $i$ represents a path from $s$ to $i$. Each state includes a resource consumption vector $R$ whose component $R_{r}$ represents the quantity of resource $r$ used along the corresponding path. Each state has an associated $\operatorname{cost} C$ and the optimal solution corresponds to a minimum cost state associated with vertex $t$. The algorithm repeatedly extends each state to generate new states. The extension of a state corresponds to appending an additional arc $(i, j)$ to a path from $s$ to $i$, obtaining a path from $s$ to $j$. This operation is repeated until all states have been extended in all feasible ways. This dynamic programming algorithm, devised for the RCSPP, can be adapted to solve the RCESPP on graphs with negative cost cycles. To this purpose Beasley and Christofides [3] proposed to add to the state an additional binary resource for each vertex $i \in \mathcal{N}$; there is only one unit available for each dummy resource and it is consumed when the correponding vertex is visited. The consumption of 
the $N$ dummy resources is indicated by a vector $S$ initialized at 0 . Note that $S$ does not keep any information about the order in which the vertices are visited. Hence in the basic exact dynamic programming algorithm for the RCESPP each state is represented by a label of the form $(S, R, C, i)$.

When a label $(S, R, C, i)$ associated with vertex $i$ is extended to generate another feasible label $\left(S^{\prime}, R^{\prime}, C^{\prime}, j\right)$ associated with vertex $j$, the resource consumption vectors and the cost are updated and the new state is checked for feasibility, as follows.

Cost. The cost is inizialized at 0 at vertex $s$ and it is updated according to the formula

$$
C^{\prime}=C-\lambda_{i} / 2+c_{i j}-\lambda_{j} / 2
$$

where $\lambda_{i}=-\lambda_{0}$ if $i=s$ and $\lambda_{j}=-\lambda_{0}$ if $j=t$.

Dummy resources. The dummy resources vector $S$ is initialized at 0 at vertex $s$ and the update rule is:

$$
S_{k}^{\prime}= \begin{cases}S_{k}+1 & k=j \\ S_{k} & k \neq j\end{cases}
$$

A state $(S, R, C, i)$ corresponds to an elementary path only if $S_{k} \leq 1 \forall k \in \mathcal{N}$.

According to the different kind of resources considered the extension rules and the feasibility test on $R$ take different forms.

Capacity. The capacity constraint is modeled by a single resource, representing the amount of capacity still available along the path. Let $q$ be the amount of resource consumed. When a vehicle leaves vertex $s$ all the resource is available, that is $q=0$. Every time a vertex is visited, $q$ is increased by the demand of that vertex. Hence the extension rule is:

$$
q^{\prime}=q+d_{j}
$$

A state $(S, q, C, i)$ is feasible only if $q \leq Q$.

Distribution and collection. In this case the capacity constraint is taken into account by two additional resources, whose consumption is indicated by $\pi$ and $\delta$. The first resource at vertex $i$ is the amount of load that the vehicle can pick-up after visiting $i$. Its consumption $\pi$ increases after every pick-up operation, because when the vehicle visits vertex $i$, it consumes $p_{i}$ units of this resource. The second resource at vertex $i$ indicates the amount of load that the vehicle can deliver after visiting $i$. This quantity is equal to the difference between the capacity $Q$ and the maximum amount of load that has been on board of the vehicle since its departure from $s$ up to $i$. Initially $Q$ units are available for the second resource and the available resource decreases each time a 
delivery operation is performed but it may decrease also after pick-up operations: the maximum amount the vehicle can deliver after visiting $i$ cannot be greater than the maximum amount it can pick-up after visiting $i$. Hence both $\pi$ and $\delta$ are initialized at 0 and the extension rule is:

$$
\begin{aligned}
& \pi^{\prime}=\pi+p_{j} \\
& \delta^{\prime}=\max \left\{\delta+d_{j}, \pi+p_{j}\right\}
\end{aligned}
$$

A state $(S, \pi, \delta, C, i)$ is feasible only if $\pi \leq Q$ and $\delta \leq Q$. Note that for the definition of $\pi$ and $\delta$ the latter condition implies the former.

Capacity and time windows. In this case the time elapsed is a consumed resource, monotonically increasing along the path. To represent the capacity constraint and the time window constraints, we need two resources, whose consumption is respectively indicated by $q$ and $\tau$ : they are the capacity and the time consumed up to the beginning of service at each vertex. Both of them are initialized at 0 and the extension rules are:

$$
\begin{aligned}
& q^{\prime}=q+d_{j} \\
& \tau^{\prime}=\max \left\{\tau+\theta_{i}+v_{i j}, a_{j}\right\}
\end{aligned}
$$

A state $(S, q, \tau, C, i)$ is feasible only if $q \leq Q$ and $\tau \leq b_{i}$.

The effectiveness of the dynamic programming algorithm heavily depends on the number of states generated. Hence it is essential to fathom feasible states which cannot lead to the optimal solution. To this purpose suitable dominance tests are always performed when states are extended, so that the algorithm records only non-dominated states. The dominance test is the following. Let $\left(S^{\prime}, R^{\prime}, C^{\prime}, i\right)$ and $\left(S^{\prime \prime}, R^{\prime \prime}, C^{\prime \prime}, i\right)$ be the labels of two states associated with vertex $i$; then $\left(S^{\prime}, R^{\prime}, C^{\prime}, i\right)$ dominates $\left(S^{\prime \prime}, R^{\prime \prime}, C^{\prime \prime}, i\right)$ only if

$$
\begin{aligned}
& S^{\prime} \leq S^{\prime \prime} \\
& R^{\prime} \leq R^{\prime \prime} \\
& C^{\prime} \leq C^{\prime \prime}
\end{aligned}
$$

and at least one of the inequalities is strict.

When the consumption of some resource is non-negative and obeys the triangle inequality, the domination rule can be made stronger, as pointed out by Feillet et al. [15]. The idea is to identify vertices which cannot be visited in any feasible extension of a given state owing to the limits on the resources. These vertices are called unreachable. When a vertex is found to be unreacheable from a given state, the consumption of the corresponding dummy resource in that state can be set to 1 , as if the vertex had already been visited. This allows the dynamic programming algorithm to identify a larger number of dominated states and to fathom them, thus reducing the computation time. We incorporated this method in all the algorithms we considered: the triangle inequality 
is certainly satisfied by the resources whose consumption occurs at the vertices, such as the RCESPP with capacity and the RCESPP with distribution and collection. The applicability to the case with time windows, where resource $\tau$ is consumed on the arcs, depends on whether the traveling times satisfy the triangle inequality or not; to apply the techniques of Feillet et al. to the time resource, we assumed $v_{i j}=c_{i j} \forall(i, j) \in \mathcal{A}$ in our tests.

The order in which the states are extended may be very important for the effectiveness of the overall algorithm. Here we consider label-correcting algorithms like those of Desrosiers et al. [12] and Feillet et al. [15]. States are explored according to the vertices they are associated with. All vertices are cyclically visited and for each vertex the algorithm extends all states that have not yet been extended. States associated with the same vertex can be sorted according to a secondary criterion, for instance according to the cost or the consumption of a certain resource. In the three cases we have considered states associated with the same vertex are sorted according to the values of $q, \pi$ and $\tau$ respectively.

Label-setting algorithms have also been proposed (see for instance Desrochers and Soumis [9]) but they require an hypothesis stronger than resource consumption monotonicity: in particular there must exists a resource whose consumption is not less than a certain known amount $\beta$ at each extension. In this case it is possible to define buckets of size $\beta$ and to mark as permanent all those labels for which the resource consumption falls in the range of the first bucket not yet extended. For a more detailed exposition of label-setting algorithms we refer the reader to Desrosiers et al. [11].

\section{Bounded bi-directional dynamic programming}

Bounded bi-directional dynamic programming has been recently introduced by Righini and Salani [22] to improve the exact dynamic programming algorithm described above. In bi-directional dynamic programming states are extended both forward from vertex $s$ to its successors and backward from vertex $t$ to its predecessors (see for instance Mingozzi et al. [21] for an application of this idea to a constrained TSP). With each vertex $i \in \mathcal{V}$ we associate forward states indicated by $\left(S^{f w}, R^{f w}, C^{f w}, i\right)$ and backward states indicated by $\left(S^{b w}, R^{b w}, C^{b w}, i\right)$. A path from $s$ to $t$ is detected each time a forward state $\left(S^{f w}, R^{f w}, C^{f w}, i\right)$ and a backward state $\left(S^{b w}, R^{b w}, C^{b w}, j\right)$ can be feasibly joined. Hereafter we describe backward extension rules and feasibility tests for backward states. Dominance tests on backward states are identical to those for forward states. We also illustrate the operation of joining forward and backward states to produce $s$ - $t$ paths and we review the idea of resource-based bounding.

\subsection{Backward extension and feasibility tests}

The backward cost $C^{b w}$ is initialized at 0 at vertex $t$ and whenever a backward state $\left(S^{b w}, R^{b w}, C^{b w}, j\right)$ is extended to a backward state $\left(S^{\prime} b w, R^{\prime} b w, C^{\prime} b w, i\right)$ the 
cost is updated according to the formula:

$$
C^{\prime} b w=C^{b w}-\lambda_{i} / 2+c_{i j}-\lambda_{j} / 2
$$

where $\lambda_{i}=-\lambda_{0}$ if $i=s$ and $\lambda_{j}=-\lambda_{0}$ if $j=t$.

The dummy resources vector $S^{b w}$ is initialized at 0 at vertex $t$ and the extension rule is:

$$
S_{k}^{\prime b w}= \begin{cases}S_{k}^{b w}+1 & k=j \\ S_{k}^{b w} & k \neq j\end{cases}
$$

A backward path is feasible only if $S_{k}^{b w} \leq 1 \forall k \in \mathcal{N}$.

Capacity. Resource consumption $q^{b w}$ in a backward state associated with vertex $j$ represents the overall demand of customers visited from $j$ (included) to $t$. The consumption $q^{b w}$ is initialized at 0 at vertex $t$. When a feasible backward path is extended along arc $(i, j)$ from a state $\left(S^{b w}, q^{b w}, C^{b w}, j\right)$ to a state $\left(S^{\prime b w}, q^{\prime b w}, C^{\prime b w}, i\right)$, the extension rule is:

$$
q^{\prime b w}=q^{b w}+d_{i}
$$

A backward path is feasible only if $q^{b w} \leq Q$.

Distribution and collection. Two resources, whose consumption is indicated by $\pi^{b w}$ and $\delta^{b w}$, are associated with each backward state. Their meaning, initialization and extension rules are symmetrical to those of forward labels: $\delta^{b w}$ indicates the amount of load delivered between $j$ and $t$ and $\pi^{b w}$ indicates the maximum overall amount of load on board of the vehicle between $j$ and $t$. When a backward path is extended along arc $(i, j)$ from a state $\left(S^{b w}, \pi^{b w}, \delta^{b w}, C^{b w}, j\right)$ to a state $\left(S^{\prime b w}, \pi^{\prime b w}, \delta^{\prime b w}, C^{\prime b w}, i\right)$, the extension rule is:

$$
\begin{aligned}
& \pi^{\prime b w}=\max \left\{\delta^{b w}+d_{i}, \pi^{b w}+p_{i}\right\} \\
& \delta^{\prime b w}=\delta^{b w}+d_{i}
\end{aligned}
$$

A backward path is feasible only if $\pi^{b w} \leq Q$ and $\delta^{b w} \leq Q$ (the former condition implies the latter).

Capacity and time windows. In the case of time windows for the sake of symmetry it is useful to define forward and backward time windows $\left[a_{i}^{f w}, b_{i}^{f w}\right]$ and $\left[a_{i}^{b w}, b_{i}^{b w}\right]$ as follows:

$$
\begin{aligned}
& a_{i}^{f w}=a_{i} \\
& b_{i}^{f w}=b_{i} \\
& a_{i}^{b w}=a_{i}+\theta_{i} \\
& b_{i}^{b w}=b_{i}+\theta_{i}
\end{aligned}
$$


The forward time window represents the range of feasible arrival times at vertex $i$, while the backward time window represents the range of feasible departure times from vertex $i$. The overall resource availability $T$ is equal to the maximum feasible arrival time at vertex $t$ that is $T=\max _{i \in \mathcal{N} \cup\{s\}}\left\{b_{i}^{f w}+\theta_{i}+v_{i t}\right\}$. The resource consumption $\tau^{b w}$ in a backward state associated with vertex $j$ represents the minimum time which must be consumed since the departure from $j$ up to the arrival at $t$. When a feasible backward path is extended along arc $(i, j)$ from a state $\left(S^{b w}, q^{b w}, \tau^{b w}, C^{b w}, j\right)$ to a state $\left(S^{\prime b w}, q^{\prime b w}, \tau^{\prime b w}, C^{\prime b w}, i\right)$, the extension rules are:

$$
\begin{aligned}
& q^{\prime b w}=q^{b w}+d_{i} \\
& \tau^{\prime b w}=\max \left\{\tau^{b w}+\theta_{j}+v_{i j}, T-b_{i}^{b w}\right\}
\end{aligned}
$$

A backward label associated with vertex $j$ is feasible only if $q^{b w} \leq Q$ and $\tau^{b w} \leq T-a_{j}^{b w}$.

\subsection{Joining forward and backward states}

Forward and backward paths must be joined together to produce complete $s$ - $t$ paths. When a forward path $\left(S^{f w}, q^{f w}, C^{f w}, i\right)$ is joined with a backward path $\left(S^{b w}, q^{b w}, C^{b w}, j\right)$ the cost of the resulting $s$ - $t$ path is equal to

$$
C^{f w}-\lambda_{i} / 2+c_{i j}-\lambda_{j} / 2+C^{b w}
$$

The join is subject to certain feasibility conditions on the resources. In particular the feasibility test on dummy resources $S$ imposes that a same vertex can not be visited by both paths.

$$
S_{k}^{f w}+S_{k}^{b w} \leq 1 \quad \forall k \in \mathcal{N}
$$

The feasibility test on problem-dependent resources $R$ imposes that for each resource the consumption in the overall $s$ - $t$ path can not exceed the overall amount of available resource. Hereafter we define the feasibility tests for each specific kind of resource constraints considered.

Capacity. The feasibility test on the capacity for joining a forward path $\left(S^{f w}, q^{f w}, C^{f w}, i\right)$ with a backward path $\left(S^{b w}, q^{b w}, C^{b w}, j\right)$ is

$$
q^{f w}+q^{b w} \leq Q
$$

Distribution and collection. The feasibility conditions to join a forward path $\left(S^{f w}, \pi^{f w}, \delta^{f w}, C^{f w}, i\right)$ with a backward path $\left(S^{b w}, \pi^{b w}, \delta^{b w}, C^{b w}, j\right)$ are:

$$
\begin{aligned}
& \pi^{f w}+\pi^{b w} \leq Q \\
& \delta^{f w}+\delta^{b w} \leq Q
\end{aligned}
$$


Capacity and time windows. The feasibility conditions to join a forward path $\left(S^{f w}, q^{f w}, \tau^{f w}, C^{f w}, i\right)$ with a backward path $\left(S^{b w}, q^{b w}, \tau^{b w}, C^{b w}, j\right)$ are:

$$
\begin{aligned}
& q^{f w}+q^{b w} \leq Q \\
& \tau^{f w}+\theta_{i}+v_{i j}+\theta_{j}+\tau^{b w} \leq T
\end{aligned}
$$

\subsection{Resource-based bounding}

Since all forward and backward states generated by the bi-directional search algorithm are tentatively joined, it is crucial to reduce their number as much as possible. To this purpose we select a critical resource, whose consumption is monotone along the paths, and we do not extend states in which at least half of the available amount of that resource has been consumed. This allows to greatly reduce the number of states generated still guaranteeing that the optimal solution will be found. Hereafter we describe how we have chosen the critical resource for each different kind of problem.

Capacity. The critical resource in this case is capacity. Forward and backward states are extended only if their associated resource consumption value, $q^{f w}$ or $q^{b w}$ respectively, is less than $Q / 2$.

Distribution and collection. In this case there are two resources; we consider as a critical resource the sum of the resource consumptions $\rho^{f w}=\pi^{f w}+\delta^{f w}$ for forward states and $\rho^{b w}=\pi^{b w}+\delta^{b w}$ for backward states and in both directions we extend only those states for which $\rho<Q$.

Capacity and time windows. In this last case we consider time as the critical resource and we extend only forward states for which $\tau^{f w}<T / 2$ and backward states for which $\tau^{b w}<T / 2$.

The combination of bi-directional search with resource-based bounding allows to solve larger instances (or the same instances in less time) than monodirectional dynamic programming; detailed experimental results are reported in [22]. In the next section we show how bi-directional search and resource-based bounding can be incorporated into dynamic programming algorithms based on state space relaxation.

We report hereafter the pseudo-code of the bounded bi-directional algorithm. We use the following symbols: $\Gamma_{i}^{f w}$ and $\Gamma_{i}^{b w}$ are the lists of forward and backward states associated with vertex $i ; \Delta_{i}^{+}$and $\Delta_{i}^{-}$are the sets of successors and predecessors of vertex $i$; $E$ is the set of vertices to be examined; Extend $d^{f w}(l, k)$ and Extend $d^{b w}(l, k)$ are respectively the forward and backward extension procedures, that extend state $l$ to vertex $k$; they check the resource constraints and produce only feasible states; $\operatorname{EFF}(\Gamma, l)$ is the procedure which inserts state $l$ into set $\Gamma$ applying the domination rules; Feasible $\left(l_{i}, l_{j}\right)$ checks the resource compatibility of forward state $l_{i}$ and backward state $l_{j}$ according to problem- 
dependent rules; Save $\left(l_{i}, l_{j}\right)$ saves the solution obtained joining the two states $l_{i}$ and $l_{j}$;

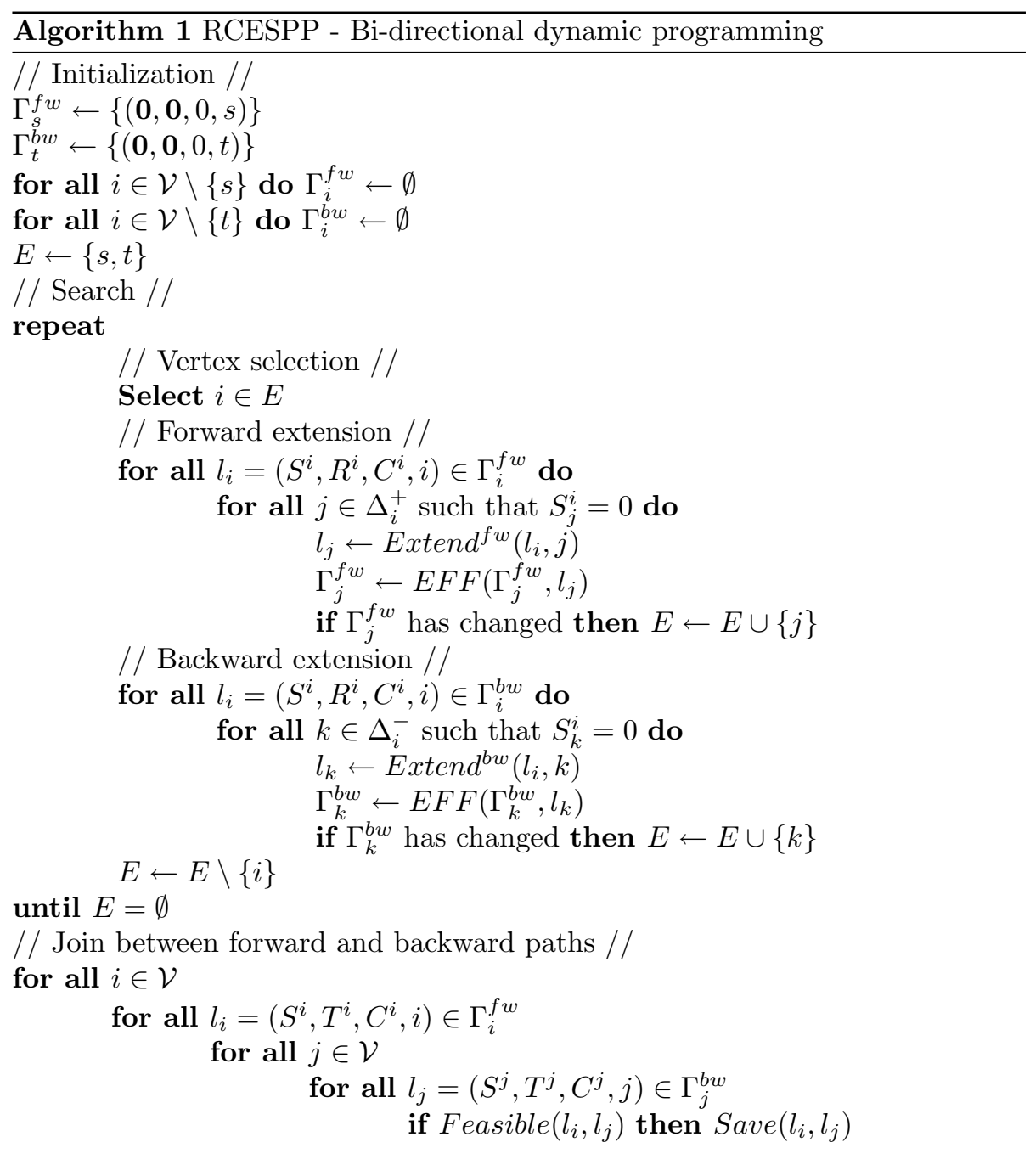

\section{State space relaxation}

State space relaxation was introduced by Christofides et al. [5] in 1981. The state space $\mathcal{S}$ explored by the dynamic programming algorithm is projected onto a lower dimensional space $\mathcal{T}$ so that each state in $\mathcal{T}$ retains the minimum cost among those of its corresponding states in $\mathcal{S}$ (assuming the objective function must be minimized). In this way the number of states to be explored is dras- 
tically reduced; the drawback is that some original state corresponding to an infeasible solution in $\mathcal{S}$ may be projected onto a state corresponding to a feasible solution in $\mathcal{T}$ and therefore the search in the relaxed state space does not guarantee to find an optimal solution but rather a lower bound.

State space relaxation has been used as a method alternative to exact optimization of the pricing problem in branch-and-price algorithms for the VRP with additional constraints (see for instance Desrochers et al. [8]): instead of the optimal value of the pricing problem, a lower bound is obtained. This allows faster convergence of the column generation algorithm at the expense of a weaker lower bound. Columns containing cycles must be eliminated through branching. Here on the contrary we focus on the use of state space relaxation for the exact optimization of the pricing problem by a branch-and-bound algorithm.

Our state space relaxation consists of mapping each state $(S, R, C, i)$ onto a new state $(\sigma, R, C, i)$, where $\sigma=\sum_{k=1}^{N} S_{k}$ represents the length of the path, that is the number of vertices visited (excluding $s$ ). Since each component of the resource consumption vector $R$ may take on a finite number of values and $\sigma$ can vary between 0 and $N$, a dynamic programming algorithm based on state space relaxation must explore only a pseudo-polynomial number of states. From the viewpoint of complexity and computing time this makes a big difference with respect to the exact dynamic programming algorithm in which vector $S$ yields an exponential number of possible states. The surrogate resource consumption $\sigma$ is initialized as 0 and it is increased by one unit each time a state is extended. Since the state does no longer keep information about the set of already visited vertices, cycles are no longer forbidden; therefore the path is guaranteed to be feasible with respect to the resource constraints but it is not guaranteed to be elementary.

In the state space relaxation algorithm the domination rule is modified as follows: a state $\left(\sigma^{\prime}, R^{\prime}, C^{\prime}, i\right)$ dominates a state $\left(\sigma^{\prime \prime}, R^{\prime \prime}, C^{\prime \prime}, i\right)$ only if

$$
\begin{aligned}
\sigma^{\prime} & \leq \sigma^{\prime \prime} \\
R^{\prime} & \leq R^{\prime \prime} \\
C^{\prime} & \leq C^{\prime \prime}
\end{aligned}
$$

and at least one of the inequalities is strict.

This state space relaxation of the RCESPP into the RCSPP can be tightened by eliminating all cycles of length two. This is easily accomplished by a duplication of the labels (see for instance Desrochers et al. [8]). Irnich and Villeneuve [19] proposed a method to eliminate cycles of length $k \geq 3$, but the computational complexity of their method dramatically increases with $k$. Hence we incorporated in our algorithms the technique to avoid cycles of length two.

The definitions above apply to both forward and backward states when bidirectional search is employed. In such case $\sigma^{f w}$ and $\sigma^{b w}$ represent respectively the number of forward extensions from $s$ and the number of backward extensions from $t$. We bound bi-directional search in the same way described above, that is on the basis of the value of a critical resource.

When bounded bi-directional search is coupled with state space relaxation the join of forward and backward paths becomes critical: both the forward path 
and the backward path to be joined may contain cycles; moreover a cycle can be produced by the join, even if the two paths are elementary. These two cases are illustrated in figure 1 . In addition there may be many different ways to join forward and backward paths providing the same solution. The former issue is addressed in the next section, where branching strategies are illustrated; the latter is addressed hereafter.
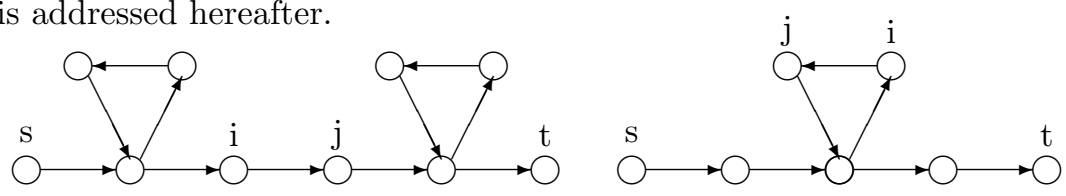

Figure 1: On the left: an $s$ - $t$ path made of non-elementary paths $s$ - $i$ and $j$ - $t$. On the right: a non-elementary $s$ - $t$ path made of elementary paths $s-i$ and $j$ - $t$.

\subsection{Paths join and solutions uniqueness}

The bounded bi-directional dynamic programming algorithm can provide duplicate solutions: consider for instance an $s$ - $t$ path including vertices $i, j$ and $k$ in this order. If the constraint on the critical resource is not tight, it is possible that forward states for vertices $i$ and $j$ and backward states for vertices $j$ and $k$ are generated. Therefore the same $s$ - $t$ path can be obtained by joining a forward state of $i$ with a backward state of $j$ as well as joining a forward state of $j$ with a backward state of $k$. This unpleasant phenomenon can be avoided with an additional test: we accept an $s$ - $t$ path only when it is produced by the join of a forward state and a backward state, for which the forward and backward consumptions of the critical resource are as close as possible to half the overall consumption for that $s$ - $t$ path, that is the two states are as close as possible to the "half way point" along the $s$ - $t$ path. Let $r^{f w}$ and $r^{b w}$ be the critical resource consumptions in forward and backward paths. Among all possible pairs of forward and backward states producing the same $s$ - $t$ path we choose the one for which $\phi=\left|r^{f w}-r^{b w}\right|$ is minimum. The test is done in constant time for each candidate pair of states, since the position closest to the "half-way point" is detected by direct comparison with the next position along the path if $r^{f w}<r^{b w}$ and with the previous position if $r^{f w}>r^{b w}$. In case of tie between two positions for which $\phi$ is minimum, we choose the one with $r^{f w}>r^{b w}$. This test guarantees that each $s$ - $t$ path is generated only once.

We report hereafter the pseudo-code of the state space relaxation algorithm where the terminology used is the same as before. In the joining step the procedure HalfWay $\left(l_{i}, l_{j}\right)$ checks if the $s$-t path obtainable joining the two states $l_{i}$ and $l_{j}$ satisfies the "half-way-point" conditions. 


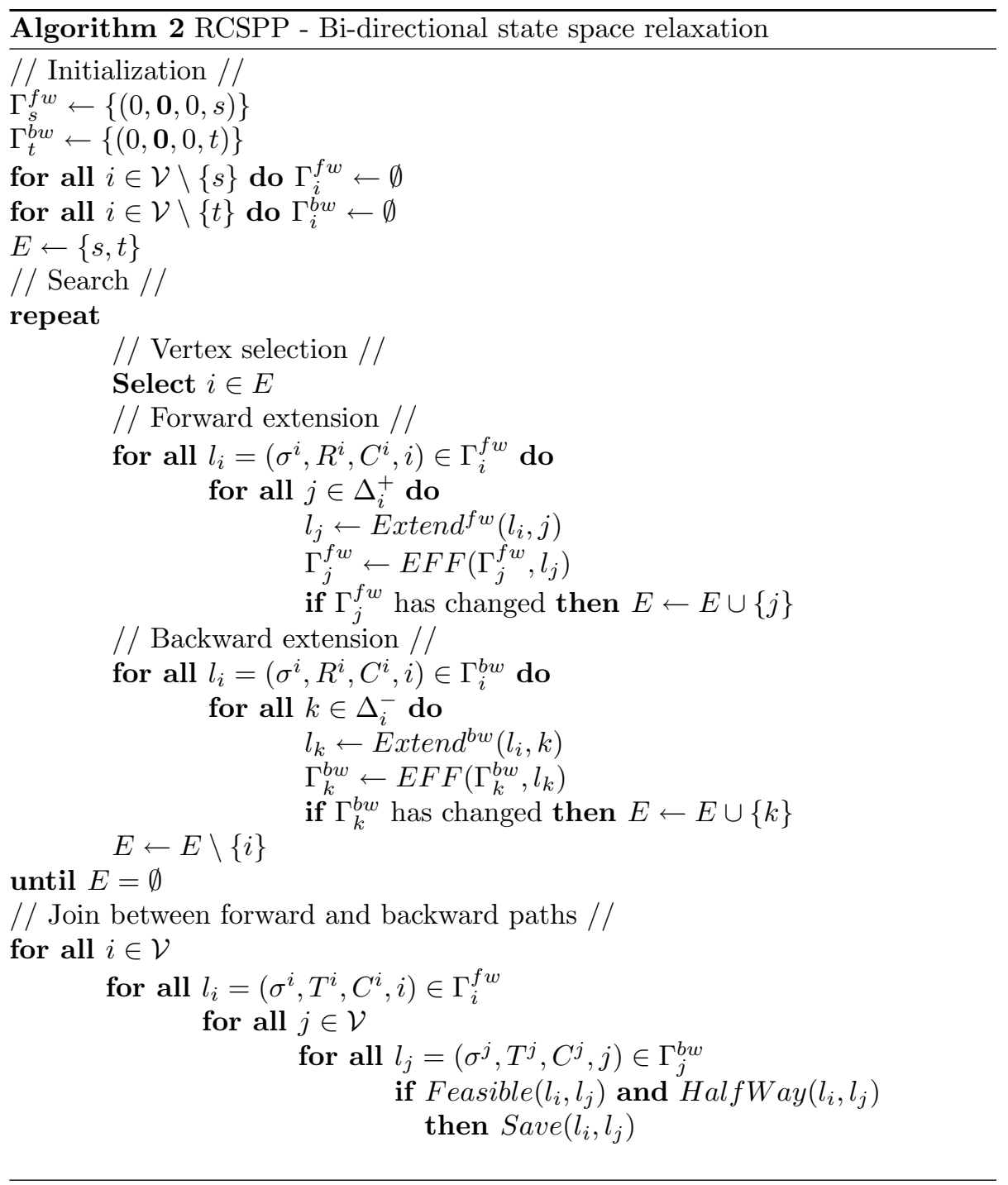

\section{Branch-and-bound}

In this section we describe a branch-and-bound algorithm which solves the RCESPP to optimality, exploiting the RCSPP lower bound given by the bounded bi-directional dynamic programming algorithm with state space relaxation. In Subsection 6.1 we describe the branching policies needed to eliminate cycles: every time the optimal solution of the RCSPP is not elementary, the current node of the search tree is replaced by children nodes in which some additional constraints are added to the RCSPP. 
Search policy. The search policy we use to explore the branch-and-bound tree is best-first, that is the open nodes of the tree are ranked according to the value of their associated lower bound and the most promising node is explored first.

Upper bounding. At each node of the branch-and-bound tree and at each iteration of the column generation algorithm a feasible solution is computed with a nearest neighbor heuristic. Starting from the depot $s$ the most convenient vertex among the feasible ones is chosen until the path reaches $t$. For a vertex to be feasible we check that no resource constraint is exceeded and the vertex have not been visited yet. At each vertex $i$ the algorithm chooses the next feasible vertex $j$ such that $j=\operatorname{argmin}_{k}\left\{c_{i k}-\lambda_{k}\right\}$.

\subsection{Branching strategies}

We present three different ways to perform branching, namely branching on cycles, branching on arcs and branching on resources. Our algorithm uses hybrid branching strategies in which all these techniques are exploited.

Branching on cycles. First we determine the minimum length cycle in the optimal RCSPP solution. Then $k$ children nodes are generated, where $k$ is the length of the cycle, that is the number of arcs traversed between two visits to the same vertex: at child node $h=0, \ldots, k-1$ we fix the first $h$ arcs of the cycle and we forbid the $h+1$-th arc. We experimentally observed that, forbidding cycles of length $2, k$ was very often equal to 3 .

Branching on arcs. This binary branching scheme consists of selecting a vertex entered or left by more than one arc in the RCSPP solution. Let $\left(i_{1}, j\right)$ and $\left(i_{2}, j\right)$ be two arcs entering vertex $j$ in the RCSPP solution. Then we partition the arcs entering $j$ into two subsets $I_{1}$ and $I_{2}$ such that $i_{1} \in I_{1}$ and $i_{2} \in I_{2}$ and we forbid all arcs in $I_{1}$ in one child node and all arcs in $I_{2}$ in the other.

Branching on resources. When the optimal solution of the RCSPP has a cycle, there exists at least one vertex $\hat{\imath}$ that is visited more than once. The branching strategy consists of adding a constraint on the quantity of critical resource consumed up to the visit of vertex $\hat{\imath}$. This idea was proposed by Gélinas et al. [16] for routing problems with time windows and it can be adapted to any problem with a critical resource whose consumption $r$ is strictly monotone along the path. Given a branching vertex $\hat{\imath}$, let $r^{\prime}$ and $r^{\prime \prime}$ the two values of resource consumption in two states associated with $\hat{\imath}$ with $r^{\prime}<r^{\prime \prime}$. Then an integer value $\bar{r}$ is chosen such that $r^{\prime}<\bar{r} \leq r^{\prime \prime}$. Two children nodes are generated imposing that the value of $r$ at vertex $\hat{\imath}$ satisfies $r \geq \bar{r}$ in one child node and $r \leq \bar{r}-1$ in the other.

It is remarkable that the dynamic programming algorithm that computes the lower bound can easily take into account the constraints imposed by all 
branching techniques. In particular when $\operatorname{arc}(i, j)$ is forbidden, it is simply deleted from the graph. The consequence of branching on the critical resource is that each vertex has an associated window $\left[a^{r}, b^{r}\right]$ of feasible values for the critical resource; when a path reaches that vertex with a critical resource consumption less than $a^{r}$, the consumption is set to $a^{r}$; when it reaches the vertex with a critical resource consumption greater than $b^{r}$, it is declared infeasible and it is discarded. This rule can be applied to both forward and backward states, with different resource windows for constraining forward and backward consumptions.

Hybrid branching. We obtained the best results when we employed hybrid branching strategies in our branch-and-bound algorithm. If either the forward path or the backward path forming the optimal RCSPP solution contain a cycle, we branch on the critical resource: we choose for branching the first vertex visited more than once which is encountered moving along the forward (resp. backward) path from $s$ to $t$ (resp. from $t$ to $s$ ); we consider $r^{\prime}$ and $r^{\prime \prime}$ as the resource consumptions at the first (resp. last) two visits of the branching vertex and we choose $\bar{r}=\left\lceil\frac{r^{\prime}+r^{\prime \prime}}{2}\right\rceil$. If the forward and the backward paths are both elementary but a cycle is generated by their join, we branch on arcs or cycles. When we branch on arcs, the branching vertex is the first vertex visited more than once which is encountered when moving along the path from the half way point forward.

We could not observe a clear domination between the hybrid branching strategies on resource/arcs and resource/cycles. In Section 8 we report on computational results obtained with each of them.

\section{Decremental state space relaxation}

The exact dynamic programming algorithm forbids multiple visits for each vertex, while the algorithm with state space relaxation does not. We pursued a compromise between these two extreme cases by the following idea: some vertices are identified as critical, according to the structure of the optimal RCSPP solution obtained with state space relaxation. Let $\Theta$ indicate the set of critical vertices at the current iteration. In the subsequent iteration the dynamic programming algorithm prevents multiple visits the vertices in $\Theta$, still allowing multiple visits to the others. This is easily accomplished by extending the state space relaxation labels with a binary vector $S_{\Theta}$ playing the same role as $S$ in exact dynamic programming. The size of $S_{\Theta}$ is however restricted only to the critical vertices. When $S_{\Theta}$ contains all the vertices the algorithm is equivalent to exact dynamic programming; when $S_{\Theta}$ is empty it is equivalent to the algorithm with state space relaxation. Therefore we indicate this algorithm by decremental state space relaxation (DSSR). The algorithm is run iteratively: every time it produces an optimal solution with cycles, the vertices visited more than once are marked as critical and the algorithm restarts. Let $\Psi$ the set of vertices visited more than once in the optimal solution computed by the DSSR 
algorithm. If $\Psi$ is not empty, then another iteration is performed with a set of critical vertices equal to $\Theta^{\prime}=\Theta \cup \Psi$. Hence the set of critical vertices is enlarged at each iteration and eventually the algorithm provides the optimal solution to the RCESPP without having recourse to branching. We report hereafter the pseudo-code of the decremental state space relaxation algorithm. where $S_{\Theta}$ is the vector of dummy resources associated to the critical vertices; procedure MultipleVisits returns the set $\Psi$ of vertices visited more than once in the current optimal path.

\section{Experimental results}

For our experiments we used the same instances as in Feillet et al. [15] and Righini and Salani [22]; they are derived from the well-known Solomon's VRPTW benchmark. For each kind of RCESPP problem we tested our algorithms on two classes of instances obtained from Solomon's instances by considering the first 50 and 100 nodes. These datasets are divided into random, clustered and random-clustered categories, according to the displacement of the customers. Instances belonging to the same dataset have the customers located in the same way and with the same delivery requests; these instances differ only for the time windows.

When solving the RCESPP with capacities we considered one instance taken from each one of the three Solomon's testsets (namely c101, r101 and rc101); we kept the original customer locations and delivery requests and we neglected the time windows. Then we derived from each original instance ten RCESPP instances with 50 nodes and ten RCESPP instances with 100 nodes, by choosing ten different values for the vehicle capacity from 10 to 100 .

For the RCESPP with distribution and collection we kept the original delivery requests and we derived the pickup requests as follows: $p_{i}=\left\lfloor 0.8 d_{i}\right\rfloor$ if $i$ is odd and $p_{i}=\left\lfloor 1.2 d_{i}\right\rfloor$ if $i$ is even. We generated ten instances with 50 nodes and ten instances with 100 nodes as before.

Finally, for the RCESPP with capacities and time windows we considered the original instances of Solomon's dataset. In addition we also defined another dataset built on the difficult Solomon's instance c_104; for each vertex $i$ we kept the original starting time of the time window, $a_{i}$, and we set the end time as follows: $b_{i}=a_{i}+(1+\gamma) \theta_{i}$ for $\gamma=0.25 * k$ and $k=0, \ldots, 24$, where $\theta_{i}$ is the given service time at vertex $i$.

For each set of instances we generated the prizes $\lambda_{i}$ as random integer variables uniformly distributed in $[0, \ldots, 20]$; we set $\lambda_{0}=0$. This data generation technique was devised by Feillet et al. [15] to have a reasonable number of negative cycles. We rounded up all the Euclidean distances between customers to integer values.

All tests were performed on a PC equipped with a PentiumIV 1.6GHz processor with $512 \mathrm{Mb}$ RAM. The algorithms were coded in ANSI-C and compiled with gcc 3.0.4. 


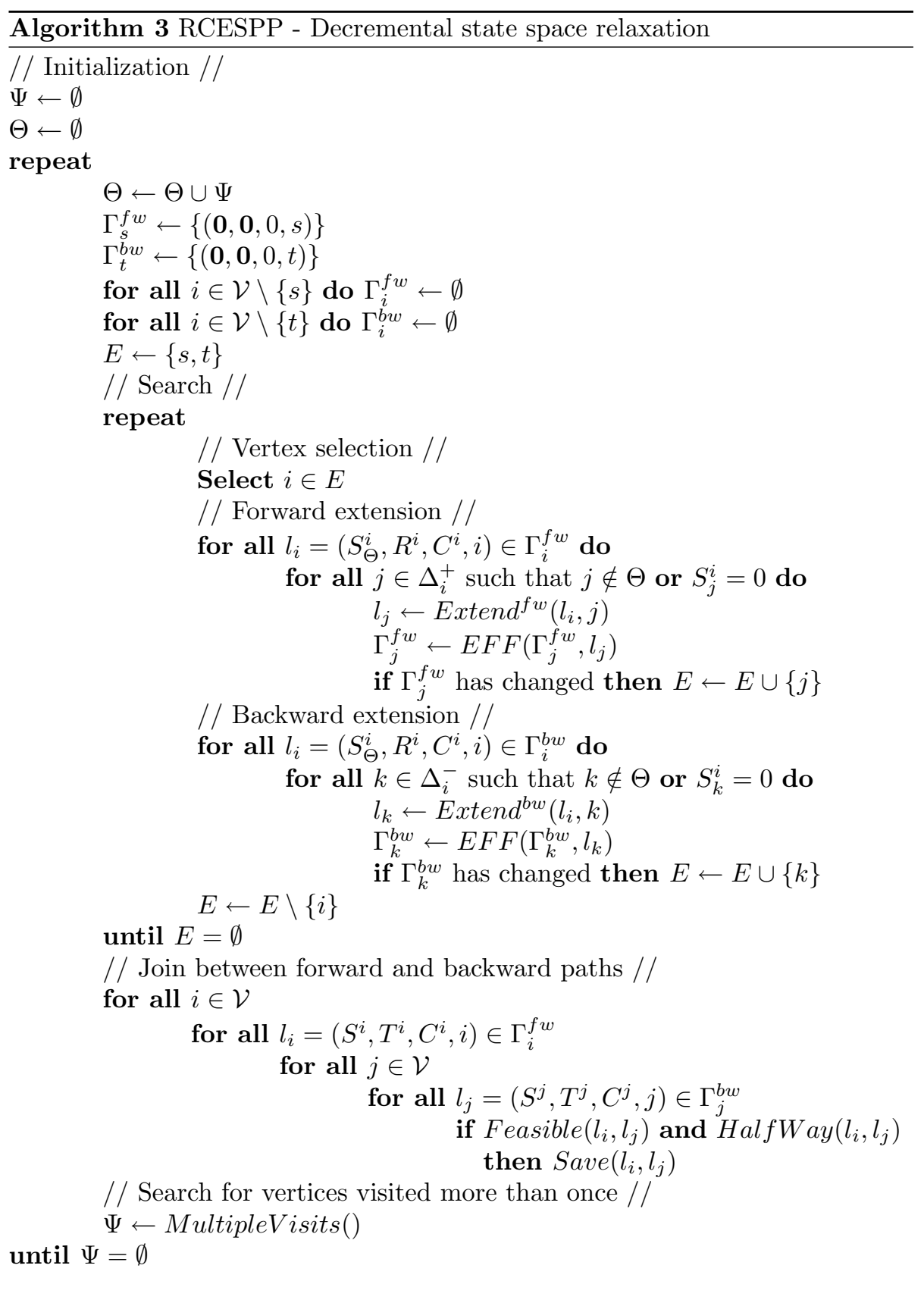

Tables 1 to 8 report on the experimental comparison between the bi-directional dynamic programming algorithm with bounds [22] (which improves on the results reported in [15]), the state space relaxation algorithm coupled with branch- 
and-bound and the decremental state space relaxation algorithm. For the bidirectional dynamic programming algorithm with bounds, named Exact D.P. in the tables, we report the total number of non-dominated labels and the computing time. For the branch-and-bound algorithm based on state space relaxation we report the total number of nodes of the search tree, the computing time and the percentage gap between the upper and the lower bounds; the reported results have been obtained with hybrid arcs/resources branching and hybrid cycles/resources branching. For the DSSR algorithm, we report the number of iterations (It), that is the number of times the bi-directional dynamic programming algorithm has been invoked, the number of critical nodes in the last iteration $(\mathrm{CN})$ and the computing time. Empty cells mean that the optimal solution has not been found within the time limit of one hour.

Capacities. Results reported in Tables 1 and 2 show that for 50 vertices instances the DSSR algorithm clearly outperforms all other algorithms on all classes of instances except for the rc-class, where exact bi-directional and bounded dynamic programming is quite fast. However these are very easy instances for all algorithms considered: the computing times are all below one second. The branch-and-bound algorithms sometimes dominate exact dynamic programming but they also fail to terminate within a reasonable computing time or even within the time-out in some cases. For 100 vertices instances the exponential growth of the computing time required by exact dynamic programming becomes evident. DSSR dramatically reduces the computing time up to two orders of magnitude. The branch-and-bound algorithms have performances similar to those of exact dynamic programming and there is no clear domination between the two hybrid branching strategies.

Distribution and collection. When solving the RCESPP with distribution and collection we obtained results similar to those above: they are reported in Tables 3 and 4 . The DSSR algorithm solved all instances in less than 340 seconds ouperforming the other algorithms and reducing the computing time by two orders of magnitude. The branch-and-bound is useful only for 100 vertices instances and the results are better for the hybrid branching on cycles and resources.

Capacities and time windows. All Solomon's instances with 50 and 100 nodes were solved by the DSSR algorithm. It should be pointed out that the most difficult instance, the c_104, has been solved within 350 seconds. For the other original Solomon's instances the branch-and-bound algorithms are not competitive, owing to the tightness and the displacement of the time windows, that often allow exact dynamic programming to go faster because the number of feasible solutions is relatively small.

Tightness of the constraints. The last two tables, 7 and 8 , show that the difficulty of a RCESPP instance does not depend only on its size but it is strongly affected by the tightness of the constraints. When time windows 
become larger and larger, the number of non-dominated states increases and so does the computing time. The growth in the number of states and computing time is due to the local nature of the time windows constraints. In these experiments the superiority of algorithms based on state space relaxation is evident. Both branch-and-bound algorithms and the DSSR algorithm solved all instances in a few seconds, whereas exact dynamic programming showed a dramatic exponential growth in computing time. When constraints are very tight, DSSR and branch-and-bound have comparable computational performances.

In spite of its simplicity the idea of decremental state space relaxation is quite effective in practice: the number of critical nodes we could observe was never greater than 15. We remark that our current implementation of the DSSR algorithm does not exploit reoptimization: information computed in the previous run could be used to speed-up successive runs. In this way the computational performances of the algorithm could be further improved.

Last but not least, the implementation of DSSR is by far easier than that of branch-and-bound.

\section{Conclusions}

We have presented and compared three different methods for the solution of the RCESPP. The first method is exact dynamic programming: though being a well-known method that has been used for nearly two decades, since the seminal work of Desrosiers et al. [12], it can be improved by new ideas, such as bi-directional search with resource-based bounding. The second method is branch-and-bound, where the lower bound is computed by dynamic programming with state space relaxation. We have outlined how bounded bi-directional search can be combined with state space relaxation and we have presented different branching strategies and their hybridization, pointing out that the lower bounding algorithm can easily handle the additional restrictions introduced by branching operations at each node of the branch-and-bound tree. The third method is a new one: decremental state space relaxation. Both exact dynamic programming and state space relaxation are special cases of this new method.

The experimental comparison of the three methods is definitely favourable to decremental state space relaxation, while no clear dominance has been observed between the other methods and not even between different hybrid branching strategies within the branch-and-bound framework. Exact dynamic programming is less robust to the constraints tightness: when the number of nondominated states grows, the computing time tends to explode very quickly.

Further improvements to the basic DSSR algorithm presented here are possible in at least two directions: first by incorporating re-optimization techniques like those of Desrochers and Soumis [10], so that each iteration of the algorithm does not restart from scratch but can re-use part of the information coming from the previous iteration; second, by guessing a clever initial subset $\Theta$ of critical nodes, instead of starting with $\Theta=\emptyset$. 
The main motivation of this study is that the RCESPP arises as a pricing subproblem in branch-and-price algorithms for the vehicle routing problem with additional constraints. A natural extension of this research is the comparison between solving the pricing problem to optimality and solving it with state space relaxation or other methods for relaxed pricing. The strategy of solving a relaxation of the pricing subproblem was adopted for instance by Agarwal et al. [1] for solving the CVRP and by Desrochers et al. [8] for solving the VRPTW, while recently Feillet et al. [15] suggested the use of exact pricing for solving the CVRPTW, by proving that tighter lower bounds (and sometimes integer optimal solutions) can be achieved at the root node by column generation with no dramatic increase in computing time. Hence the trade-off between saving computing time and improving the lower bound tightness definitely deserves further investigation and it will be subject of future research. Preliminary experiments on the CVRPTW show that a column generation algorithm in which exact pricing is done via DSSR gives the same lower bounds of Feillet et al. at the root node in only a fraction of the time, in particular for Solomon's instances of classes "c" and "rc". Although we cannot claim that the comparison analyzed in this paper can be directly transferred to the choice between exact pricing and relaxed pricing, we conjecture that the experiments reported here can give useful suggestions about the trade-off between the quality of the lower bound and the computing time required to compute it, depending on the kind of resource constraints and their tightness.

\section{Acknowledgments}

We are grateful to Dominique Feillet for providing us his code. We also acknowledge the support of ACSU - Associazione Cremasca Studi Universitari to the Operations Research Laboratory of our department, where this research was done.

\section{References}

[1] Agarwal Y., Mathur K., Salkin H.M., A set-partitioning-based exact algorithm for the vehicle routing problem, Networks 19 (1989) 731-749

[2] Ahuja R.K., Magnanti T.L., Orlin J.B., Network flows, Prentice Hall 1993

[3] Beasley J.E., Christofides N., An algorithm for the resource constrained shortest path problem, Networks 19 (1989) 379-394

[4] Bramel J., Simchi-Levi D., Set-covering-based algorithms for the capacitated VRP, in The vehicle routing problem, Toth P., Vigo D. eds., SIAM Monographs on Discrete Mathematics and Applications, 2002

[5] Christofides N., Mingozzi A., Toth P., State-space relaxation procedures for the computation of bounds to routing problems Networks 11, 145-164 (1981). 
[6] Cordeau J.F., Desaulniers G., Desrosiers J., Solomon M.M., Soumis F., VRP with time windows, in The vehicle routing problem, Toth P., Vigo D. eds., SIAM Monographs on Discrete Mathematics and Applications, 2002

[7] Desrochers M., An algorithm for the shortest path problem with resource constraints, Cahiers du GERAD G-88-27, University of Montreal (1988)

[8] Desrochers M., Desrosiers J., Solomon M., A new optimization algorithm for the vehicle routing problem with time windows, Operations Research 40 (1992) 342-354

[9] Desrochers M., Soumis F., A generalized permanent labelling algorithm for the shortest path problem with time windows, INFOR 26, 191-212 (1988)

[10] Desrochers M., Soumis F., A reoptimization algorithm for the shortest path problem with time windows, European Journal of Operational Research 35 (1988) 242-254

[11] Desrosiers J., Dumas Y., Solomon M., Soumis F., Time constrained routing and scheduling in Network Routing, Ball M.O. et al. eds., Handbooks in Operations Research and Management Science, Elsevier Science 1995

[12] Desrosiers J., Pelletier P., Soumis F., Plus court chemin avec contraintes d'horaires, RAIRO 17, 357-377 (1983)

[13] Dror M., Note on the complexity of the shortest path models for column generation in VRPTW, Operations Research 42 (1994) 977-978

[14] Dumitresu I., Boland N., Improved preprocessing, labeling and scaling algorithms for the weight-constrained shortest path problem, Networks 42, 135-153 (2003)

[15] Feillet D., Dejax P., Gendreau M., Gueguen C., An exact algorithm for the elementary shortest path problem with resource constraints: application to some vehicle routing problems, Networks 44, 216-229 (2004)

[16] Gélinas S., Desrochers M., Desrosiers J., Solomon M.M., A new branching strategy for time constrained routing problems with application to backhauling Cahiers du GERAD G-92-13, HEC Montréal, 1992.

[17] Handler G.Y., Zang I., A dual algorithm for the constrained shortest path problem, Networks 10 (1980) 293-310

[18] Irnich S., Desaulniers G., Shortest path problems with resource constraints, Cahier du GERAD G-2004-11, Université de Montréal, 2004

[19] Irnich S., Villeneuve D., The shortest path problem wiht resource constraints and $k$-cycle elimination for $k \geq 3$, Cahiers du GERAD G-2003-55, HEC Montréal, 2003 
[20] Kohl N., Desrosiers J., Madsen O.B.G., Solomon M.M., Soumis F., 2-path cuts for the vehicle routing problem with time windows Transportation Science 33 (1999) 101-116

[21] Mingozzi A., Bianco L., Ricciardelli S., Dynamic programming strategies for the traveling salesman problem with time window and precedence constraints, Operations Research 45 (1997) 365-377

[22] Righini G., Salani M., Symmetry helps: bounded bi-directional dynamic programming for the elementary shortest path problem with resource constraints, Note del Polo - Ricerca 66, DTI - Università degli Studi di Milano, 2004 (submitted for publication) 
Table 1: RCESPP with capacity - 50 vertices

\begin{tabular}{|c|c|c|c|c|c|c|c|c|c|c|c|}
\hline \multirow{2}{*}{$\begin{array}{r}\text { Instance } \\
\text { Name }\end{array}$} & \multicolumn{2}{|c|}{ Exact D.P. } & \multicolumn{3}{|c|}{ B\&B Res+Arcs } & \multicolumn{3}{|c|}{ B\&B Res+Cycles } & \multicolumn{3}{|c|}{ DSSR } \\
\hline & Labels & Time & Nodes & Time & $(\%)$ & Nodes & Time & (\%) & It & $\mathrm{CN}$ & Time \\
\hline c_50_01 & 56 & 0.00 & 1 & 0.00 & - & 1 & 0.00 & - & 1 & 0 & 0.00 \\
\hline c_50_02 & 268 & 0.00 & 1 & 0.00 & - & 1 & 0.00 & - & 1 & 0 & 0.00 \\
\hline c_50_03 & 692 & 0.00 & 7 & 0.01 & - & 1 & 0.02 & - & 1 & 0 & 0.00 \\
\hline c_50_04 & 2574 & 0.03 & 19 & 0.08 & - & 10 & 0.05 & - & 3 & 2 & 0.02 \\
\hline c_50_05 & 4692 & 0.07 & 11 & 0.05 & - & 22 & 0.09 & - & 2 & 2 & 0.01 \\
\hline c_50_06 & 15236 & 0.91 & 87 & 0.48 & - & 116 & 0.91 & - & 3 & 3 & 0.04 \\
\hline c_50_07 & 23394 & 1.75 & 35 & 0.24 & - & 52 & 0.33 & - & 2 & 3 & 0.02 \\
\hline C_50_08 & 75026 & 20.35 & 315 & 3.19 & - & 124 & 1.81 & - & 5 & 7 & 0.25 \\
\hline c_50_09 & 101128 & 33.24 & 673 & 5.80 & - & 224 & 1.78 & - & 4 & 8 & 0.18 \\
\hline c_50_10 & 331402 & 394.97 & 3919 & 44.56 & - & 3039 & 53.34 & - & 5 & 10 & 1.82 \\
\hline r_50_01 & 62 & 0.00 & 1 & 0.00 & - & 1 & 0.00 & - & 1 & 0 & 0.00 \\
\hline r_50_02 & 210 & 0.01 & 1 & 0.01 & - & 1 & 0.00 & - & 1 & 0 & 0.00 \\
\hline r_50_03 & 525 & 0.01 & 7 & 0.04 & - & 1 & 0.00 & - & 3 & 3 & 0.02 \\
\hline r_50_04 & 1250 & 0.02 & 7 & 0.06 & - & 1 & 0.00 & - & 2 & 3 & 0.02 \\
\hline r_50_05 & 2418 & 0.05 & 591 & 6.64 & - & 7 & 0.09 & - & 6 & 7 & 0.17 \\
\hline r_50_06 & 4570 & 0.11 & 271 & 4.49 & - & 83 & 1.18 & - & 4 & 6 & 0.12 \\
\hline r_50_07 & 7874 & 0.24 & 6009 & 90.34 & - & 122 & 1.12 & - & 2 & 4 & 0.06 \\
\hline r_50_08 & 13590 & 0.60 & 3149 & 38.75 & - & 95 & 0.98 & - & 5 & 11 & 0.48 \\
\hline r_50_09 & 22800 & 1.49 & 191 & 6.12 & - & 17 & 0.53 & - & 4 & 9 & 0.40 \\
\hline r_50_10 & 36838 & 3.87 & 59 & 1.16 & - & 7 & 0.26 & - & 3 & 8 & 0.34 \\
\hline rc_50_01 & 44 & 0.00 & 1 & 0.00 & - & 1 & 0.00 & - & 1 & $\overline{0}$ & 0.00 \\
\hline rc_50_02 & 124 & 0.00 & 1 & 0.00 & - & 1 & 0.00 & - & 1 & 0 & 0.00 \\
\hline rc_50_03 & 268 & 0.00 & 11 & 0.02 & - & 1 & 0.01 & - & 4 & 3 & 0.00 \\
\hline rc_50_04 & 560 & 0.01 & 725 & 1.58 & - & 73 & 0.17 & - & 5 & 4 & 0.02 \\
\hline rc_50_05 & 800 & 0.01 & 1573 & 4.21 & - & 173 & 0.42 & - & 4 & 4 & 0.02 \\
\hline rc_50_06 & 1551 & 0.03 & 73573 & 562.40 & - & 1774 & 8.29 & - & 7 & 8 & 0.09 \\
\hline rc_50_07 & 1774 & 0.04 & 3417 & 19.66 & - & 84 & 0.50 & - & 4 & 7 & 0.05 \\
\hline rc_50_08 & 3217 & 0.08 & 239467 & - & 6.25 & 10505 & 49.19 & - & 7 & 11 & 0.20 \\
\hline rc_50_09 & 3322 & 0.09 & 29021 & 348.97 & - & 960 & 7.68 & - & 6 & 11 & 0.20 \\
\hline rc_50_10 & 5864 & 0.19 & 254931 & - & 2.0 & 16679 & 156.19 & - & 6 & 11 & 0.27 \\
\hline
\end{tabular}


Table 2: RCESPP with capacity - 100 vertices

\begin{tabular}{|c|c|c|c|c|c|c|c|c|c|c|c|}
\hline \multirow{2}{*}{$\begin{array}{r}\text { Instance } \\
\text { Name }\end{array}$} & \multicolumn{2}{|c|}{ Exact D.P. } & \multicolumn{3}{|c|}{ B\&B Res+Arcs } & \multicolumn{3}{|c|}{ B\&B Res+Cycles } & \multicolumn{3}{|c|}{ DSSR } \\
\hline & Labels & Time & Nodes & Time & $(\%)$ & Nodes & Time & $(\%)$ & It & $\mathrm{CN}$ & Time \\
\hline c_100_01 & 106 & 0.00 & 1 & 0.00 & - & 1 & 0.00 & - & 1 & 0 & 0.00 \\
\hline c_100_02 & 559 & 0.01 & 1 & 0.01 & - & 1 & 0.01 & - & 1 & 0 & 0.00 \\
\hline c_100_03 & 1456 & 0.03 & 7 & 0.04 & - & 1 & 0.02 & - & 2 & 1 & 0.03 \\
\hline c_100_04 & 5900 & 0.19 & 19 & 0.26 & - & 10 & 0.15 & - & 3 & 2 & 0.06 \\
\hline c_100_05 & 11546 & 0.44 & 43 & 0.36 & - & 22 & 0.31 & - & 3 & 4 & 0.07 \\
\hline c_100_06 & 45138 & 6.60 & 193 & 5.19 & - & 699 & 16.31 & - & 4 & 5 & 0.21 \\
\hline c_100_07 & 75698 & 13.78 & 65 & 1.84 & - & 99 & 2.65 & - & 3 & 6 & 0.18 \\
\hline c_100_08 & 310651 & 276.88 & 1275 & 77.50 & - & 517 & 30.53 & - & 6 & 10 & 1.34 \\
\hline c_100_09 & 4799333 & 520.82 & 8125 & 326.11 & - & 862 & 32.71 & - & 6 & 14 & 2.02 \\
\hline c_100_10 & - & - & 11465 & 627.08 & - & 12076 & 910.73 & - & 6 & 13 & 7.68 \\
\hline r_100_01 & 266 & 0.00 & 15 & 0.04 & - & 10 & 0.04 & - & 3 & 3 & 0.02 \\
\hline r_100_02 & 2120 & 0.06 & 459 & 3.61 & - & 225 & 1.45 & - & 2 & 4 & 0.06 \\
\hline r_100_03 & 11866 & 0.70 & 5337 & 126.87 & - & 776 & 15.03 & - & 4 & 5 & 0.59 \\
\hline r_100_04 & 53668 & 8.37 & 7639 & 306.49 & - & 1819 & 69.68 & - & 4 & 7 & 2.80 \\
\hline r_100_05 & 215976 & 104.41 & 81677 & - & 7.3 & 4464 & 198.93 & - & 3 & 5 & 2.87 \\
\hline r_100_06 & 764476 & 1300.33 & 51755 & - & 9.5 & 13209 & 1282.61 & - & 4 & 8 & 34.64 \\
\hline r_100_07 & - & - & 31121 & - & 11.2 & 29182 & - & 1.1 & 5 & 10 & 143.63 \\
\hline r_100_08 & - & - & 12548 & - & 25.4 & 18741 & - & 6.3 & 5 & 11 & 281.62 \\
\hline r_100_09 & - & - & 6912 & - & 51.1 & 12549 & - & 18.9 & 3 & 10 & 303.34 \\
\hline r_100_10 & - & - & 2551 & - & 67.4 & 6118 & - & 43.2 & 3 & 10 & 319.68 \\
\hline rc_100_01 & 90 & 0.00 & 1 & 0.00 & - & 1 & 0.00 & - & 1 & 0 & 0.00 \\
\hline rc_100_02 & 636 & 0.01 & 1 & 0.01 & - & 1 & 0.00 & - & 1 & 0 & 0.00 \\
\hline rc_100_03 & 1732 & 0.04 & 31 & 0.33 & - & 10 & 0.13 & - & 1 & 0 & 0.00 \\
\hline rc_100_04 & 5706 & 0.23 & 7 & 0.20 & - & 10 & 0.27 & - & 2 & 1 & 0.07 \\
\hline rc_100_05 & 12561 & 0.69 & 1669 & 71.45 & - & 64 & 2.35 & - & 4 & 4 & 0.29 \\
\hline rc_100_06 & 29786 & 2.80 & 71 & 2.52 & - & 60 & 3.01 & - & 3 & 4 & 0.35 \\
\hline rc_100_07 & 60499 & 9.74 & 4403 & 376.85 & - & 752 & 59.22 & - & 4 & 5 & 0.92 \\
\hline rc_100_08 & 124752 & 37.46 & 5735 & 353.00 & - & 254 & 25.00 & - & 4 & 7 & 1.77 \\
\hline rc_100_09 & 237652 & 130.20 & 739 & 109.08 & - & 391 & 28.76 & - & 3 & 5 & 1.40 \\
\hline rc_100_10 & 459269 & 470.24 & 25055 & - & 3.7 & 7385 & 1191.96 & - & 5 & 10 & 7.33 \\
\hline
\end{tabular}


Table 3: RCESPP with distribution and collection - 50 vertices

\begin{tabular}{|c|c|c|c|c|c|c|c|c|c|c|c|}
\hline Instance & \multicolumn{2}{|c|}{ Exact D.P. } & \multicolumn{3}{|c|}{ B\&B Res+Arcs } & \multicolumn{3}{|c|}{ B\&B Res+Cycles } & \multicolumn{3}{|c|}{ DSSR } \\
\hline Name & Labels & Time & Nodes & Time & $(\%)$ & Nodes & Time & $(\%)$ & It & $\mathrm{CN}$ & Time \\
\hline c_50_01 & 26 & 0.00 & 1 & 0.00 & - & 1 & 0.00 & - & 1 & 0 & 0.00 \\
\hline c_50_02 & 159 & 0.00 & 1 & 0.00 & - & 1 & 0.00 & - & 1 & 0 & 0.00 \\
\hline c_50_03 & 554 & 0.01 & 1 & 0.02 & - & 1 & 0.01 & - & 2 & 1 & 0.00 \\
\hline c_50_04 & 1751 & 0.02 & 7 & 0.02 & - & 10 & 0.04 & - & 2 & 1 & 0.01 \\
\hline c_50_05 & 4675 & 0.07 & 15 & 0.07 & - & 22 & 0.10 & - & 2 & 2 & 0.02 \\
\hline c_50_06 & 11311 & 0.41 & 27 & 0.17 & - & 122 & 0.94 & - & 3 & 3 & 0.05 \\
\hline c_50_07 & 24006 & 1.72 & 17 & 0.16 & - & 53 & 0.48 & - & 2 & 3 & 0.03 \\
\hline c_50_08 & 51401 & 7.95 & 83 & 0.90 & - & 91 & 1.44 & - & 5 & 7 & 0.47 \\
\hline c_50_09 & 110354 & 35.46 & 69 & 0.88 & - & 207 & 2.47 & - & 3 & 5 & 0.19 \\
\hline c_50_10 & 233478 & 165.21 & 119 & 3.56 & - & 129 & 3.66 & - & 5 & 10 & 2.20 \\
\hline r_50_01 & 59 & 0.00 & 1 & 0.00 & - & 1 & 0.00 & - & 1 & 0 & 0.00 \\
\hline r_50_02 & 188 & 0.00 & 5 & 0.02 & - & 7 & 0.02 & - & 2 & 1 & 0.01 \\
\hline r_50_03 & 486 & 0.01 & 1 & 0.01 & - & 1 & 0.01 & - & 3 & 3 & 0.01 \\
\hline r_50_04 & 1113 & 0.02 & 1 & 0.03 & - & 1 & 0.03 & - & 2 & 3 & 0.02 \\
\hline r_50_05 & 2085 & 0.04 & 11 & 0.14 & - & 7 & 0.08 & - & 5 & 6 & 0.13 \\
\hline r_50_06 & 3882 & 0.10 & 17 & 0.28 & - & 45 & 0.62 & - & 3 & 5 & 0.07 \\
\hline r_50_07 & 6986 & 0.23 & 3 & 0.07 & - & 3 & 0.08 & - & 2 & 4 & 0.06 \\
\hline r_50_08 & 12138 & 0.51 & 71 & 0.97 & - & 122 & 1.13 & - & 4 & 7 & 0.19 \\
\hline r_50_09 & 20384 & 1.23 & 13 & 0.42 & - & 19 & 0.59 & - & 3 & 7 & 0.21 \\
\hline r_50_10 & 33107 & 3.15 & 5 & 0.21 & - & 5 & 0.21 & - & 3 & 7 & 0.25 \\
\hline $\begin{array}{l}\text { rc_50_01 } \\
\end{array}$ & 24 & 0.00 & 1 & 0.00 & - & 1 & 0.00 & - & 1 & 0 & 0.00 \\
\hline rc_50_02 & 83 & 0.00 & 1 & 0.00 & - & 1 & 0.00 & - & 1 & 0 & 0.01 \\
\hline rc_50_03 & 199 & 0.01 & 1 & 0.01 & - & 1 & 0.01 & - & 3 & 2 & 0.01 \\
\hline rc_50_04 & 397 & 0.01 & 55 & 0.12 & - & 73 & 0.14 & - & 5 & 4 & 0.02 \\
\hline rc_50_05 & 764 & 0.02 & 171 & 0.51 & - & 114 & 0.28 & - & 4 & 5 & 0.03 \\
\hline rc_50_06 & 1108 & 0.02 & 3321 & 19.86 & - & 1156 & 5.01 & - & 6 & 7 & 0.09 \\
\hline rc_50_07 & 1817 & 0.03 & 397 & 3.00 & - & 233 & 1.40 & - & 6 & 7 & 0.09 \\
\hline rc_50_08 & 2546 & 0.05 & 595 & 6.41 & - & 405 & 4.07 & - & 4 & 7 & 0.05 \\
\hline rc_50_09 & 3435 & 0.10 & 4363 & 63.94 & - & 666 & 8.13 & - & 7 & 9 & 0.22 \\
\hline rc_50_10 & 4998 & 0.15 & 12045 & 210.69 & - & 6551 & 79.95 & - & 6 & 11 & 0.24 \\
\hline
\end{tabular}


Table 4: RCESPP with distribution and collection - 100 vertices

\begin{tabular}{|c|c|c|c|c|c|c|c|c|c|c|c|}
\hline \multirow{2}{*}{$\begin{array}{r}\text { Instance } \\
\text { Name }\end{array}$} & \multicolumn{2}{|c|}{ Exact D.P. } & \multicolumn{3}{|c|}{ B\&B Res+Arcs } & \multicolumn{3}{|c|}{ B\&B Res+Cycles } & \multicolumn{3}{|c|}{ DSSR } \\
\hline & Labels & Time & Nodes & Time & $(\%)$ & Nodes & Time & $(\%)$ & It & $\mathrm{CN}$ & Time \\
\hline c_100_01 & 48 & 0.00 & 1 & 0.00 & - & 1 & 0.00 & - & 1 & 0 & 0.00 \\
\hline c_100_02 & 328 & 0.00 & 1 & 0.00 & - & 1 & 0.00 & - & 1 & 0 & 0.02 \\
\hline c_100_03 & 1179 & 0.02 & 1 & 0.00 & - & 1 & 0.00 & - & 2 & 1 & 0.02 \\
\hline c_100_04 & 3829 & 0.08 & 7 & 0.10 & - & 10 & 0.13 & - & 3 & 2 & 0.06 \\
\hline c_100_05 & 11112 & 0.41 & 15 & 0.26 & - & 22 & 0.40 & - & 3 & 4 & 0.10 \\
\hline c_100_06 & 30823 & 2.62 & 15 & 0.54 & - & 597 & 17.26 & - & 4 & 5 & 0.25 \\
\hline c_100_07 & 76548 & 12.72 & 35 & 1.24 & - & 101 & 3.67 & - & 3 & 6 & 0.27 \\
\hline c_100_08 & 197386 & 87.88 & 175 & 6.56 & - & 192 & 12.57 & - & 6 & 10 & 2.17 \\
\hline c_100_09 & 509042 & 516.42 & 239 & 15.83 & - & 884 & 58.01 & - & 5 & 11 & 2.34 \\
\hline c_100_10 & - & - & 737 & 89.37 & - & 952 & 11.27 & - & 7 & 15 & 20.64 \\
\hline r_100_01 & 253 & 0.00 & 1 & 0.00 & - & 1 & 0.00 & - & 1 & 0 & 0.00 \\
\hline r_100_02 & 1948 & 0.06 & 801 & 7.47 & - & 222 & 1.52 & - & 2 & 4 & 0.06 \\
\hline r_100_03 & 10874 & 0.68 & 7275 & 186.84 & - & 831 & 16.90 & - & 4 & 5 & 0.64 \\
\hline r_100_04 & 49258 & 7.34 & 15297 & 790.61 & - & 1814 & 69.59 & - & 3 & 5 & 1.34 \\
\hline r_100_05 & 189041 & 84.00 & 40991 & 3503.40 & - & 5242 & 357.35 & - & 3 & 5 & 3.71 \\
\hline r_100_06 & 676338 & 1040.25 & 42932 & - & 5.0 & 14627 & 1074.52 & - & 4 & 8 & 39.63 \\
\hline r_100_07 & - & - & 36124 & - & 8.9 & 24782 & - & 1.4 & 5 & 10 & 180.41 \\
\hline r_100_08 & - & - & 19733 & - & 22.3 & 30764 & - & 12.5 & 4 & 10 & 217.66 \\
\hline r_100_09 & - & - & 8153 & - & 45.5 & 11489 & - & 22.0 & 3 & 10 & 337.47 \\
\hline r_100_10 & - & - & 3559 & - & 62.8 & 4025 & - & 61.0 & 3 & 10 & 337.43 \\
\hline rc_100_01 & 67 & 0.00 & 1 & 0.00 & - & 1 & 0.00 & - & 1 & 0 & 0.00 \\
\hline rc_100_02 & 501 & 0.01 & 1 & 0.00 & - & 1 & 0.00 & - & 1 & 0 & 0.00 \\
\hline rc_100_03 & 1422 & 0.04 & 7 & 0.10 & - & 10 & 0.13 & - & 2 & 1 & 0.03 \\
\hline rc_100_04 & 4540 & 0.17 & 7 & 0.19 & - & 10 & 0.26 & - & 2 & 1 & 0.07 \\
\hline rc_100_05 & 10790 & 0.55 & 27 & 1.16 & - & 61 & 2.25 & - & 4 & 4 & 0.30 \\
\hline rc_100_06 & 25657 & 2.29 & 23 & 1.18 & - & 54 & 2.98 & - & 3 & 4 & 0.33 \\
\hline rc_100_07 & 52378 & 7.73 & 801 & 74.78 & - & 736 & 58.96 & - & 4 & 5 & 0.97 \\
\hline rc_100_08 & 107414 & 28.62 & 361 & 44.60 & - & 242 & 24.13 & - & 3 & 5 & 1.11 \\
\hline rc_100_09 & 207049 & 99.56 & 235 & 23.30 & - & 389 & 29.55 & - & 3 & 5 & 1.55 \\
\hline rc_100_10 & - & - & 5671 & 971.28 & - & 7162 & 1207.45 & - & 4 & 8 & 6.11 \\
\hline
\end{tabular}


Table 5: RCESPP with capacity and time windows - 50 vertices

\begin{tabular}{|c|c|c|c|c|c|c|c|c|c|c|c|}
\hline \multirow{2}{*}{$\begin{array}{r}\text { Instance } \\
\text { Name }\end{array}$} & \multicolumn{2}{|c|}{ Exact D.P. } & \multicolumn{3}{|c|}{ B\&B Res+Arcs } & \multicolumn{3}{|c|}{ B\&B Res+Cycles } & \multicolumn{3}{|c|}{ DSSR } \\
\hline & Labels & Time & Nodes & Time & $(\%)$ & Nodes & Time & $(\%)$ & It & $\mathrm{CN}$ & Time \\
\hline c101_50 & 323 & 0.01 & 1 & 0.00 & - & 1 & 0.00 & - & 1 & 0 & 0.00 \\
\hline c102_50 & 3026 & 0.14 & 15 & 0.19 & - & 12 & 0.19 & - & 2 & 2 & 0.12 \\
\hline c103_50 & 30736 & 10.25 & 2533 & 127.32 & - & 966 & 27.74 & - & 4 & 6 & 14.06 \\
\hline c104_50 & - & - & 35781 & - & 1.2 & 24785 & 1835.47 & - & 4 & 11 & 344.65 \\
\hline c105_50 & 435 & 0.02 & 1 & 0.02 & - & 1 & 0.02 & - & 1 & 0 & 0.01 \\
\hline c106_50 & 359 & 0.01 & 1 & 0.03 & - & 1 & 0.03 & - & 1 & 0 & 0.02 \\
\hline c107_50 & 504 & 0.02 & 1 & 0.04 & - & 1 & 0.04 & - & 1 & 0 & 0.02 \\
\hline c108_50 & 736 & 0.04 & 1 & 0.04 & - & 1 & 0.04 & - & 1 & 0 & 0.04 \\
\hline c109_50 & 2031 & 0.15 & 81 & 1.90 & - & 78 & 1.69 & - & 8 & 11 & 1.35 \\
\hline r101_50 & 121 & 0.00 & 1 & 0.00 & - & 1 & 0.00 & - & 1 & 0 & 0.00 \\
\hline r102_50 & 596 & 0.02 & 5 & 0.05 & - & 4 & 0.04 & - & 4 & 5 & 0.08 \\
\hline r103_50 & 2322 & 0.06 & 817 & 9.83 & - & 103 & 1.07 & - & 3 & 4 & 0.27 \\
\hline r104_50 & 15441 & 0.66 & 83 & 2.46 & - & 264 & 7.08 & - & 3 & 5 & 0.77 \\
\hline r105_50 & 238 & 0.01 & 1 & 0.01 & - & 1 & 0.01 & - & 1 & 0 & 0.0 \\
\hline r106_50 & 818 & 0.02 & 13 & 0.14 & - & 9 & 0.08 & - & 4 & 6 & 0.18 \\
\hline r107_50 & 2784 & 0.08 & 15435 & 248.92 & - & 268 & 3.10 & - & 4 & 5 & 0.47 \\
\hline r108_50 & 16457 & 0.75 & 5 & 0.15 & - & 5 & 0.15 & - & 2 & 4 & 0.48 \\
\hline r109_50 & 584 & 0.02 & 1 & 0.02 & - & 1 & 0.02 & - & 1 & 0 & 0.02 \\
\hline r110_50 & 1600 & 0.04 & 1 & 0.04 & - & 1 & 0.04 & - & 1 & 0 & 0.04 \\
\hline r111_50 & 2289 & 0.07 & 43 & 0.73 & - & 46 & 0.66 & - & 3 & 5 & 0.33 \\
\hline r112_50 & 3987 & 0.12 & 1 & 0.05 & - & 1 & 0.05 & - & 1 & 0 & 0.05 \\
\hline rc101_50 & 270 & 0.00 & 1 & 0.00 & - & 1 & 0.00 & - & 1 & 0 & 0.01 \\
\hline rc102_50 & 906 & 0.01 & 17 & 0.13 & - & 17 & 0.13 & - & 3 & 3 & 0.09 \\
\hline rc103_50 & 3509 & 0.07 & 112 & 3.45 & - & 6168 & 90.88 & - & 5 & 11 & 0.95 \\
\hline rc104_50 & 8801 & 0.28 & 258 & 7.65 & - & 312 & 8.31 & - & 7 & 15 & 4.77 \\
\hline rc105_50 & 937 & 0.01 & 63 & 0.48 & - & 60 & 0.32 & - & 3 & 4 & 0.11 \\
\hline rc106_50 & 889 & 0.01 & 71 & 0.52 & - & 676 & 4.64 & - & 4 & 7 & 0.16 \\
\hline rc107_50 & 3525 & 0.07 & 29567 & 653.53 & - & 13452 & 195.907 & - & 6 & 9 & 0.83 \\
\hline rc108_50 & 10166 & 0.21 & 34205 & 1143.65 & - & 58476 & 1474.19 & - & 6 & 13 & 2.12 \\
\hline
\end{tabular}


Table 6: RCESPP with capacity and time windows - 100 vertices

\begin{tabular}{|c|c|c|c|c|c|c|c|c|c|c|c|}
\hline \multirow{2}{*}{$\begin{array}{r}\text { Instance } \\
\text { Name }\end{array}$} & \multicolumn{2}{|c|}{ Exact D.P. } & \multicolumn{3}{|c|}{ B\&B Res+Arcs } & \multicolumn{3}{|c|}{ B\&B Res+Cycles } & \multicolumn{3}{|c|}{ DSSR } \\
\hline & Labels & Time & Nodes & Time & $(\%)$ & Nodes & Time & $(\%)$ & It & $\mathrm{CN}$ & Time \\
\hline c101_100 & 679 & 0.06 & 1 & 0.02 & - & 1 & 0.02 & - & 1 & 0 & 0.02 \\
\hline c102_100 & 11839 & 2.99 & 15 & 0.89 & - & 12 & 0.67 & - & 2 & 2 & 0.63 \\
\hline c103_100 & 123804 & 133.56 & 2539 & 507.78 & - & 3075 & 485.68 & - & 5 & 9 & 40.15 \\
\hline c104_100 & - & - & 17553 & - & 17.8 & 23402 & - & 16.5 & 4 & 11 & 311.44 \\
\hline c105_100 & 915 & 0.11 & 1 & 0.06 & - & 1 & 0.06 & - & 1 & 0 & 0.06 \\
\hline c106_100 & 1159 & 0.16 & 1 & 0.07 & - & 1 & 0.07 & - & 1 & 0 & 0.07 \\
\hline c107_100 & 1058 & 0.16 & 1 & 0.08 & - & 1 & 0.08 & - & 1 & 0 & 0.08 \\
\hline c108_100 & 1690 & 0.33 & 1 & 0.17 & - & 1 & 0.17 & - & 1 & 0 & 0.17 \\
\hline c109_100 & 4608 & 1.28 & 111 & 14.18 & - & 101 & 12.66 & - & 8 & 13 & 9.19 \\
\hline r101_100 & 452 & 0.01 & 1 & 0.00 & - & 1 & 0.00 & - & 1 & $\overline{0}$ & 0.00 \\
\hline r102_100 & 14792 & 2.21 & 4203 & 438.82 & - & 1003 & 96.25 & - & 3 & 6 & 21.69 \\
\hline r103_100 & 135575 & 95.73 & 377 & 128.71 & - & 252 & 61.10 & - & 4 & 7 & 159.74 \\
\hline r104_100 & 655858 & 1242.56 & 4531 & - & 0.9 & 1945 & 1013.04 & - & 3 & 5 & 78.32 \\
\hline r105_100 & 1161 & 0.06 & 1 & 0.03 & - & 1 & 0.03 & - & 1 & 0 & 0.03 \\
\hline r106_100 & 22970 & 5.52 & 26059 & - & 3.4 & 3344 & 467.29 & - & 4 & 7 & 71.60 \\
\hline r107_100 & 138027 & 100.83 & 1417 & 717.37 & - & 732 & 232.91 & - & 4 & 12 & 335.98 \\
\hline r108_100 & 570910 & 891.81 & 1593 & 1098.05 & - & 1451 & 611.61 & - & 3 & 5 & 146.58 \\
\hline r109_100 & 3504 & 0.37 & 49 & 3.39 & - & 49 & 3.45 & - & 3 & 5 & 2.93 \\
\hline r110_100 & 25063 & 4.89 & 307 & 69.25 & - & 406 & 77.55 & - & 3 & 6 & 19.31 \\
\hline r111_100 & 69890 & 25.86 & 2669 & 866.34 & - & 361 & 129.24 & - & 3 & 7 & 53.16 \\
\hline r112_100 & 394702 & 647.36 & 2167 & 2227.74 & - & 665 & 385.00 & - & 3 & 9 & 340.61 \\
\hline rc101_100 & 955 & 0.03 & 1 & 0.02 & - & 1 & 0.02 & - & 1 & 0 & 0.02 \\
\hline rc102_100 & 5384 & 0.30 & 17 & 1.12 & - & 21 & 1.34 & - & 4 & 4 & 3.17 \\
\hline rc103_100 & 38308 & 6.01 & 861 & 110.78 & - & 6494 & 587.39 & - & 4 & 8 & 35.37 \\
\hline rc104_100 & 232961 & 148.73 & 607 & 194.68 & - & 1054 & 203.36 & - & 4 & 8 & 102.56 \\
\hline rc105_100 & 2964 & 0.15 & 7 & 0.43 & - & 13 & 0.67 & - & 4 & 6 & 1.14 \\
\hline rc106_100 & 2574 & 0.12 & 31 & 1.85 & - & 12 & 0.56 & - & 3 & 3 & 1.01 \\
\hline rc107_100 & 10505 & 0.72 & 87 & 8.50 & - & 27 & 2.79 & - & 4 & 6 & 3.65 \\
\hline rc108_100 & 45430 & 6.75 & 239 & 32.34 & - & 143 & 12.43 & - & 3 & 5 & 4.84 \\
\hline
\end{tabular}


Table 7: RCESPP with capacity and time windows - Instance c_104, 50 vertices

\begin{tabular}{|r|rr|rrr|rrr|rr|r|}
\hline Instance & \multicolumn{2}{|c|}{ Exact D.P. } & \multicolumn{2}{|c|}{ B\&B Res+Arcs } & \multicolumn{2}{|c|}{ B\&B Res+Cycles } & \multicolumn{3}{c|}{ DSSR } \\
Name & Labels & Time & Nodes & Time & $(\%)$ & Nodes & Time & $(\%)$ & It & CN & Time \\
\hline c104_50_01 & 59 & 0.00 & 1 & 0.01 & - & 1 & 0.01 & - & 1 & 0 & 0.01 \\
c104_50_02 & 126 & 0.00 & 1 & 0.01 & - & 1 & 0.01 & - & 1 & 0 & 0.01 \\
c104_50_03 & 200 & 0.01 & 1 & 0.01 & - & 1 & 0.01 & - & 1 & 0 & 0.01 \\
c104_50_04 & 206 & 0.01 & 1 & 0.01 & - & 1 & 0.01 & - & 1 & 0 & 0.01 \\
c104_50_05 & 208 & 0.01 & 1 & 0.01 & - & 1 & 0.01 & - & 1 & 0 & 0.01 \\
c104_50_06 & 269 & 0.01 & 1 & 0.02 & - & 1 & 0.02 & - & 1 & 0 & 0.02 \\
c104_50_07 & 475 & 0.01 & 1 & 0.02 & - & 1 & 0.02 & - & 1 & 0 & 0.02 \\
c104_50_08 & 586 & 0.04 & 1 & 0.02 & - & 1 & 0.02 & - & 1 & 0 & 0.02 \\
c104_50_09 & 730 & 0.05 & 1 & 0.02 & - & 1 & 0.02 & - & 1 & 0 & 0.02 \\
c104_50_10 & 804 & 0.06 & 1 & 0.02 & - & 1 & 0.02 & - & 1 & 0 & 0.02 \\
c104_50_11 & 1440 & 0.09 & 1 & 0.03 & - & 1 & 0.03 & - & 1 & 0 & 0.03 \\
c104_50_12 & 2292 & 0.21 & 1 & 0.03 & - & 1 & 0.03 & - & 1 & 0 & 0.03 \\
c104_50_13 & 3771 & 0.43 & 1 & 0.04 & - & 1 & 0.04 & - & 1 & 0 & 0.04 \\
c104_50_14 & 4031 & 0.53 & 19 & 0.24 & - & 13 & 0.18 & - & 2 & 3 & 0.14 \\
c104_50_15 & 5508 & 0.74 & 13 & 0.22 & - & 9 & 0.12 & - & 3 & 4 & 0.49 \\
c104_50_16 & 9411 & 2.10 & 39 & 0.88 & - & 12 & 0.25 & - & $: 3$ & 4 & 0.53 \\
c104_50_17 & 18579 & 5.02 & 45 & 1.09 & - & 15 & 0.35 & - & 3 & 4 & 0.60 \\
c104_50_18 & 21738 & 6.54 & 145 & 3.53 & - & 76 & 1.66 & - & 3 & 4 & 0.61 \\
c104_50_19 & 25638 & 9.25 & 77 & 2.43 & - & 64 & 1.41 & - & 3 & 5 & 1.20 \\
c104_50_20 & 36762 & 23.16 & 85 & 2.02 & - & 91 & 2.35 & - & 4 & 6 & 2.11 \\
c104_50_21 & 81804 & 73.19 & 179 & 5.95 & - & 144 & 3.99 & - & 4 & 6 & 2.69 \\
c104_50_22 & 105756 & 110.25 & 199 & 5.35 & - & 91 & 2.52 & - & 3 & 6 & 2.31 \\
c104_50_23 & 126645 & 149.81 & 255 & 11.00 & - & 214 & 9.34 & - & 8 & 11 & 17.45 \\
c104_50_24 & 157915 & 275.93 & 276 & 19.7 & - & 238 & 17.98 & - & 5 & 7 & 13.43 \\
c104_50_25 & 323641 & 1016.98 & 321 & 18.36 & - & 381 & 19.83 & - & 4 & 7 & 13.31 \\
\hline
\end{tabular}

Table 8: RCESPP with capacity and time windows - Instance c_104, 100 vertices

\begin{tabular}{|c|c|c|c|c|c|c|c|c|c|c|c|}
\hline \multirow{2}{*}{$\begin{array}{r}\text { Instance } \\
\text { Name }\end{array}$} & \multicolumn{2}{|c|}{ Exact D.P. } & \multicolumn{3}{|c|}{ B\&B Res+Arcs } & \multicolumn{3}{|c|}{ B\&B Res+Cycles } & \multicolumn{3}{|c|}{ DSSR } \\
\hline & Labels & Time & Nodes & Time & $(\%)$ & Nodes & Time & $(\%)$ & It & $\mathrm{CN}$ & Time \\
\hline c104_100_01 & 160 & 0.01 & 1 & 0.04 & - & 1 & 0.04 & - & 1 & 0 & 0.04 \\
\hline c104_100_02 & 227 & 0.01 & 1 & 0.05 & - & 1 & 0.05 & - & 1 & 0 & 0.05 \\
\hline c104_100_03 & 368 & 0.02 & 1 & 0.06 & - & 1 & 0.06 & - & 1 & 0 & 0.06 \\
\hline c104_100_04 & 415 & 0.04 & 1 & 0.07 & - & 1 & 0.07 & - & 1 & 0 & 0.07 \\
\hline c104_100_05 & 427 & 0.05 & 1 & 0.07 & - & 1 & 0.07 & - & 1 & 0 & 0.07 \\
\hline c104_100_06 & 523 & 0.06 & 1 & 0.08 & - & 1 & 0.08 & - & 1 & 0 & 0.08 \\
\hline c104_100_07 & 895 & 0.11 & 1 & 0.08 & - & 1 & 0.08 & - & 1 & 0 & 0.08 \\
\hline c104_100_08 & 1153 & 0.19 & 1 & 0.09 & - & 1 & 0.09 & - & 1 & 0 & 0.09 \\
\hline c104_100_09 & 1393 & 0.29 & 1 & 0.09 & - & 1 & 0.09 & - & 1 & 0 & 0.09 \\
\hline c104_100_10 & 1557 & 0.37 & 1 & 0.09 & - & 1 & 0.09 & - & 1 & 0 & 0.09 \\
\hline c104_100_11 & 2655 & 0.59 & 1 & 0.11 & - & 1 & 0.11 & - & 1 & 0 & 0.11 \\
\hline c104_100_12 & 4504 & 1.24 & 1 & 0.12 & - & 1 & 0.12 & - & 1 & 0 & 0.12 \\
\hline c104_100_13 & 7336 & 2.37 & 1 & 0.11 & - & 1 & 0.11 & - & 1 & 0 & 0.11 \\
\hline c104_100_14 & 8457 & 3.30 & 33 & 1.76 & - & 11 & 0.74 & - & 3 & 3 & 1.49 \\
\hline c104_100_15 & 10932 & 4.49 & 65 & 3.24 & - & 21 & 1.05 & - & 2 & 3 & 1.26 \\
\hline c104_100_16 & 19133 & 9.75 & 249 & 15.12 & - & 23 & 1.62 & - & 2 & 3 & 1.56 \\
\hline c104_100_17 & 39114 & 23.83 & 211 & 20.02 & - & 21 & 1.60 & - & 2 & 3 & 1.65 \\
\hline c104_100_18 & 53650 & 39.11 & 75 & 8.64 & - & 30 & 3.54 & - & 2 & 3 & 1.65 \\
\hline c104_100_19 & 66165 & 56.43 & 45 & 5.85 & - & 30 & 2.99 & - & 4 & 6 & 6.32 \\
\hline c104_100_20 & 91825 & 110.71 & 59 & 4.66 & - & 42 & 2.04 & - & 4 & 5 & 7.84 \\
\hline c104_100_21 & 197498 & 336.64 & 67 & 7.29 & - & 48 & 4.26 & - & 4 & 5 & 8.79 \\
\hline c104_100_22 & 315475 & 702.11 & 89 & 10.78 & - & 59 & 5.94 & - & 3 & 6 & 7.54 \\
\hline c104_100_23 & 437113 & 1169.71 & 53 & 7.94 & - & 53 & 7.60 & - & 7 & 9 & 32.06 \\
\hline c104_100_24 & 547902 & 1945.73 & 141 & 31.02 & - & 143 & 33.46 & - & 5 & 7 & 29.87 \\
\hline c104_100_25 & - & - & 187 & 47.05 & - & 17 & 44.32 & - & 4 & 7 & 27.74 \\
\hline
\end{tabular}

